Homology, Homotopy and Applications, vol.9(2), 2007, pp.95-100

\title{
ON HIGHER NIL GROUPS OF GROUP RINGS
}

\author{
DANIEL JUAN-PINEDA
}

(communicated by Charles Weibel)

\begin{abstract}
Let $G$ be a finite group and $\mathbb{Z}[G]$ its integral group ring. We prove that the nil groups $N^{j} K_{2}(\mathbb{Z}[G])$ do not vanish for all $j \geqslant 1$ and for a large class of finite groups. We obtain from this that the iterated nil groups $N^{j} K_{i}(\mathbb{Z}[G])$ are also nonzero for all $i \geqslant 2, j \geqslant i-1$.
\end{abstract}

\section{Introduction}

Let $\Gamma$ be a discrete group and $\mathbb{Z}[\Gamma]$ its integral group ring. The Farrell-Jones Isomorphism Conjecture [13] predicts that the algebraic $K$-theory groups $K_{i}(\mathbb{Z}[\Gamma])$ may be computed from homological information of $\Gamma$ and the algebraic $K$-theory of group rings $R[V]$, where $V$ runs over the virtually cyclic subgroups of $\Gamma$. When the Farrell-Jones Isomorphism Conjecture holds there have been explicit examples like $[\mathbf{8}, \mathbf{9}, \mathbf{2 0}]$ and it has been the case that these computations may even be reduced further to the case where $V$ runs over the finite subgroups of $\Gamma[\mathbf{9 , 2 0}]$; see Section 4 for a precise formulation. The groups that prevent such reductions are the nil groups of the group rings of finite subgroups of $\Gamma$; see H. Bass [5] for definitions. In this paper we show that, in principle, such reductions cannot be achieved for $K_{i}(\mathbb{Z}[\Gamma])$ for $i>1$. Our main result is the following:

Theorem 1.1. Let $G$ be a nontrivial finite cyclic group or a split extension of a nontrivial finite cyclic group. Then

$$
N^{j} K_{i}(\mathbb{Z}[G]) \neq 0 \text { for all } i \geqslant 2 \text { and } j \geqslant i-1 .
$$

The above is related to an old question by H. Bass [6, Prob. VI]. We conjecture that this nonvanishing result must hold for every finite group.

We thank Chuck Weibel for his valuable comments and explanations during the preparation of this work.

Research partially supported by CONACyT and DGAPA research grants, and the ICMS, Edinburgh.

Received October 5, 2006, revised May 28, 2007; published on July 30, 2007.

2000 Mathematics Subject Classification: 19A31, 19C99, 19 D35.

Key words and phrases: $K$-theory, nil groups.

Copyright (C) 2007, International Press. Permission to copy for private use granted. 
Homology, Homotopy and Applications, vol. 9(2), 2007

\section{Preliminaries}

Let $R$ be an associative ring with unity and $K_{n}(R)$ its $n$th algebraic $K$-theory; cf. D. Quillen $[\mathbf{2 1}]$. Let $G$ be a group and $R[G]$ its corresponding group ring. It is natural to compare the $K$-theory of $R$ to that of its polynomial ring $R[t]$. This leads to the definition of the nil groups: Let $\epsilon: R[t] \rightarrow R$ be the augmentation map, induced by evaluating at $t=0$. The $i$ th nil group of $R$ is defined as

$$
N K_{i}(R)=\operatorname{ker}\left(K_{i}(R[t]) \stackrel{\epsilon_{*}}{\longrightarrow} K_{i}(R)\right) .
$$

By iterating this to polynomial rings in more variables we get the iterated nil groups $N^{j} K_{i}(R)=N\left(N^{j-1} K_{i}(R)\right), j>1$. These nil $K$-groups have geometric significance as they occur as obstructions to geometric problems; see for example [11]. We are interested in the study of these nil $K$-groups in the case when $R=\mathbb{Z}[G]$ and $G$ is a finite group.

Recall that when $R$ is a regular ring, it follows that $N K_{i}(R)=0$ for all $i$; cf. [21]. The rings $\mathbb{Z}[G]$ are never regular when $G$ is a finite group. However, some vanishing results are available: if $G$ is a finite group of square-free order, then $N K_{i}(\mathbb{Z}[G])=0$ for $i=0,1$; see Harmon $[\mathbf{1 6}]$. In fact, $N K_{i}(\mathbb{Z}[G])=0$ for all $i \leqslant-1$ and any finite group $G ;[\mathbf{5}$, XII, 10.2]. It has been conjectured by W.C. Hsiang that this last property should hold for any integral group ring. This has been verified for large classes of groups as a consequence of the Farrell-Jones Conjecture in [18].

Another instance in which these nil $K$-groups appear naturally is in the setup of the Farrell-Jones Conjecture; see Section 4.

\section{Nonvanishing results}

In [15], Guin-Waléry and Loday proved that $N K_{2}\left(\mathbb{Z}\left[C_{p}\right]\right) \cong x \mathbb{Z} / p[x]$, and is generated by Dennis-Stein symbols $\left\langle(1-\sigma) x^{j},\left(1+\sigma+\cdots \sigma^{p-1}\right)\right\rangle$, where $C_{p}$ stands for the finite cyclic group of prime order $p$ and generated by $\sigma$.

Theorem 3.1. Let $C_{n}$ be a cyclic group of finite order $n \geqslant 2$. Then $N K_{2}\left(\mathbb{Z}\left[C_{n}\right]\right)$ $\neq 0$.

Proof. As there is a split summand $C_{n} \rightarrow C_{p^{r}}$, we may assume that $n=p^{r}$ for some prime $p$ and integer $r \geqslant 2$. Let $\sigma$ be a generator of $C_{n}$. Observe that as $(1-\sigma)\left(1+\sigma+\cdots+\sigma^{p^{r}-1}\right)=1-\sigma^{p^{r}}=0$ in $\mathbb{Z}\left[C_{n}\right]$, the symbol

$$
\left\langle\left(1+\sigma+\cdots+\sigma^{p^{r}-1}\right) x,(1-\sigma)\right\rangle
$$

is a well-defined Dennis-Stein symbol in $K_{2}\left(\mathbb{Z}\left[C_{n}\right][x]\right)$. We will prove that this is not trivial as long as $r \geqslant 1$. Let

$$
\varphi: K_{2}\left(\mathbb{Z}\left[C_{n}\right][x]\right) \rightarrow K_{2}\left(\mathbb{F}_{p}\left[C_{n}\right][x]\right)
$$

be induced from $\bmod p$ reduction. We see that

$$
\mathbb{F}_{p}\left[C_{n}\right][x] \cong \mathbb{F}_{p}[\varepsilon] / \varepsilon^{p^{r}}[x] \cong \mathbb{F}_{p}[\varepsilon, x] /\left(\varepsilon^{p^{r}}\right),
$$

and, under the above identifications, $\sigma$ is taken to $1-\varepsilon$. On the other hand, let $I$ be the ideal generated by $\varepsilon$ in $\mathbb{F}_{p}[\varepsilon, x] /\left(\varepsilon^{p^{r}}\right)$; thus $\left(\mathbb{F}_{p}[\varepsilon, x] /\left(\varepsilon^{p^{r}}\right)\right) / I \cong \mathbb{F}_{p}[x]$. From the 
long exact sequence associated to the pair $\left(\mathbb{F}_{p}[\varepsilon, x] /\left(\varepsilon^{p^{r}}\right), I\right)$, the fact that $K_{3}\left(\mathbb{F}_{p}[x]\right)$ is isomorphic to $K_{3}\left(\mathbb{F}_{p}\right)$, which is a group of order prime to $p$, and by $[\mathbf{1 9}$, Corollary 2.7], the first group below is a $p$-group, so we have a monomorphism

$$
K_{2}\left(\mathbb{F}_{p}[\varepsilon, x] /\left(\varepsilon^{p^{r}}\right), I\right) \hookrightarrow K_{2}\left(\mathbb{F}_{p}[\varepsilon, x] /\left(\varepsilon^{p^{r}}\right)\right) .
$$

Now observe that

$$
\begin{aligned}
& \varphi\left(\left\langle\left(1+\sigma+\cdots+\sigma^{p^{r}-1}\right) x,(1-\sigma)\right\rangle\right) \\
& =\left\langle\left(1+(1-\varepsilon)+(1-\varepsilon)^{2}+\cdots+(1-\varepsilon)^{p^{r}-1}\right) x, \varepsilon\right\rangle \\
& =\left\langle\varepsilon^{p^{r}-1} x, \varepsilon\right\rangle .
\end{aligned}
$$

This element $\left\langle\varepsilon^{p^{r}-1} x, \varepsilon\right\rangle$ as an element of $K_{2}\left(\mathbb{F}_{p}[\varepsilon, x] /\left(\varepsilon^{p^{r}}\right)\right)$ comes from the relative group $K_{2}\left(\mathbb{F}_{p}[\varepsilon, x] /\left(\varepsilon^{p^{r}}\right), I\right)$ and is a generator of order $p$ in the relative group by the computations of W. van der Kallen and J. Stienstra in [19, Corollary 2.7]. Finally, observe that this element really is in $N K_{2}\left(\mathbb{F}_{p}[\varepsilon] /\left(\varepsilon^{p^{r}}\right),(\varepsilon)\right) \cong N K_{2}\left(\mathbb{F}_{p}[\varepsilon] /\left(\varepsilon^{p^{r}}\right)\right)$.

C. Weibel, proved (see [23, Application III.3.4.2]) that for any ring if $N^{s} K_{i}(R)$ $=0$, it follows that $N^{j} K_{i}(R)=0$ for $j=1,2, \ldots, s-1$. As a corollary we have

Corollary 3.2. Let $C_{n}$ be a nontrivial finite cyclic group, then

$$
N^{j} K_{2}\left(\mathbb{Z}\left[C_{n}\right]\right) \neq 0, \text { for all } j \geqslant 1 \text {. }
$$

This contrasts with $N K_{1}\left(\mathbb{Z}\left[C_{n}\right]\right)$ where it is known $[\mathbf{7}]$ that

$$
N K_{1}\left(\mathbb{Z}\left[C_{n}\right]\right)=0 \text { if and only if } n \text { is square-free. }
$$

By the fundamental theorem in algebraic $K$-theory, the $N K_{2}$ terms are direct summands in the group $K_{2}\left(\mathbb{Z}\left[C_{n} \times T^{s}\right]\right)$, where $T^{s}$ is the free abelian group of rank $s$, and $s \geqslant 1$. Moreover, by [12] we see that if $N K_{2}$ is nontrivial then it is not finitely generated. Thus we have the following

Corollary 3.3. Let $C_{n}$ be a nontrivial finite cyclic group and $T^{s}$ a free abelian group of rank $s, s \geqslant 1$. Then $K_{2}\left(\mathbb{Z}\left[C_{n} \times T^{s}\right]\right)$ is not finitely generated.

A ring $R$ is called $K_{n}$-regular if $N^{j} K_{n}(R)=0$ for all $j \geqslant 1$. T. Vorst proved in [22, Proposition 2.1] that if $N^{2} K_{n}(R)=0$ then $N K_{n-1}(R)=0$. Hence, if $R$ is such that $N K_{2}(R) \neq 0$ it follows that $N^{j} K_{i}(R) \neq 0$ for $j \geqslant i-1$ and for all $i>2$. From this we have the following corollary:

Corollary 3.4. Let $C_{n}$ and $T^{s}$ be a finite cyclic group of order $n, n \geqslant 2$, and the free abelian group of rank $s \geqslant 1$, respectively. Then

1. $N^{j} K_{i}\left(\mathbb{Z}\left[C_{n}\right]\right) \neq 0$ for all $i \geqslant 2$ and all $j \geqslant i-1$,

2. $K_{i}\left(\mathbb{Z}\left[C_{n} \times T^{s}\right]\right)$ is not finitely generated for all $i \geqslant 2$, and $s \geqslant i-1$. 
The results in the previous section immediately give the proof of Theorem 1.1.

Proof. The cyclic case is Theorem 3.1. Let $r: G \rightarrow C$ be a split surjection onto a cyclic group $C$. Then the splitting $s: C \rightarrow G$ induces an injection $N^{j} K_{i}(\mathbb{Z}[C]) \hookrightarrow$ $N^{j} K_{i}(\mathbb{Z}[G]) ;$ thus our result follows from Theorem 3.1 and the above.

\section{Examples}

Our results contrast with those for lower $K$-theory where it is known that $N K_{-i}(\mathbb{Z}[G])=0$ for all $i \geqslant 1$ and all finite groups $G$; see H. Bass [5, XII, 10.2]. On the other hand, if $G$ is a finite group of square-free order it is known that $N K_{i}(\mathbb{Z}[G])=0$ for $i=0,1$; see $[\mathbf{1 6}]$. The following examples show consequences of our results for infinite groups of geometric relevance.

We begin by recalling some terminology: a group $V$ is called virtually cyclic if it contains a cyclic group of finite index. It follows that either $V$ is finite or it contains a unique maximal normal finite subgroup $F$ such that either:

1. $V / F$ is infinite cyclic or

2. $V / F$ is infinite dihedral.

We call $V$ orientable if the first case above holds and is nonorientable in the second case; see [17].

Example 4.1. Let $G$ be a word hyperbolic group in the sense of Gromov; see [14]. Assume that all finite subgroups of $G$ satisfy the hypotheses of Theorem 1.1 and that the only infinite virtually cyclic subgroups of $G$ are of the form $F \times \mathbb{Z}$, where $F$ is a finite subgroup of $G$. Let $R$ be an associative ring, and $\mathbb{K}_{R}$ be the algebraic $K$ theory spectrum defined in [10]. Given $X$ a $G$-CW-complex, write $H_{*}^{G}\left(X ; \mathbb{K}_{R}\right)$ for the associated equivariant homology theory applied to $X$. This theory is such that for any $H \leqslant G, H_{*}^{G}\left(G / H ; \mathbb{K}_{R}\right)$ is naturally isomorphic to the algebraic $K$-groups $K_{*}(R H)$. The following description of this equivariant homology for hyperbolic groups is found in [17, Corollary 19 and Remark 7]: For any word hyperbolic group $G$ as above, there is an isomorphism

$$
H_{n}^{G}\left(\underline{\underline{\mathcal{E}}} G ; \mathbb{K}_{\mathbb{Z}}\right) \cong H_{n}^{G}\left(\underline{\mathcal{E}} G ; \mathbb{K}_{\mathbb{Z}}\right) \oplus \bigoplus_{\operatorname{Conj}(V)} N K_{n}(\mathbb{Z}[\operatorname{fin}(V)]) \oplus N K_{n}(\mathbb{Z}[\operatorname{fin}(V)]),
$$

where

- $\operatorname{Conj}(V)$ denotes representatives of conjugacy classes of maximal infinite virtually cyclic subgroups of $G$,

- $\operatorname{fin}(V)$ is the finite maximal subgroup of $V$,

- the spaces $\underline{\underline{\mathcal{E}}} G$ and $\underline{\mathcal{E}} G$ are universal spaces for actions with virtually cyclic and finite isotropy respectively.

As a corollary of the above, we have:

Corollary 4.2. Let $G$ be as in Example 4.1; then $H_{2}^{G}\left(\underline{\underline{\mathcal{E}}} G ; \mathbb{K}_{\mathbb{Z}}\right)$ is not finitely generated. 
Remark 4.3. It has been announced by A. Bartels, H. Reich and W. Lück that the Farrell-Jones Isomorphism Conjecture in $K$-theory holds for hyperbolic groups [3]; hence $H_{n}^{G}\left(\underline{\underline{\mathcal{E}}} G ; \mathbb{K}_{\mathbb{Z}}\right)$ is really $K_{n}(\mathbb{Z}[G])$.

Example 4.4. Let $\Gamma$ be a discrete group. Assume that $\Gamma$ has nontrivial torsion, that the finite subgroups of $\Gamma$ satisfy the hypotheses of Theorem 1.1, and that the FarrellJones Isomorphism Conjecture holds for $\mathbb{Z}[\Gamma][\mathbf{1 3}]$. Then, the algebraic $K$-theory of $\mathbb{Z}[\Gamma]$ is isomorphic to the generalized equivariant homology theory (Example 4.1):

$$
H_{n}^{\Gamma}\left(\underline{\underline{\mathcal{E}}} \Gamma ; \mathbb{K}_{\mathbb{Z}}\right)
$$

where $\underline{\underline{\mathcal{E}}} \Gamma$ denotes the universal space for actions with virtually cyclic isotropy.

On the other hand, we may take $H_{n}^{\Gamma}\left(\underline{\mathcal{E}} \Gamma ; \mathbb{K}_{\mathbb{Z}}\right)$, where $\underline{\mathcal{E}} \Gamma$ denotes the universal space for actions with finite isotropy. There is a natural map induced by inclusions

$$
\mathcal{A}: H_{n}^{\Gamma}\left(\underline{\mathcal{E}} \Gamma ; \mathbb{K}_{\mathbb{Z}}\right) \rightarrow H_{n}^{\Gamma}\left(\underline{\underline{\mathcal{E}}} \Gamma ; \mathbb{K}_{\mathbb{Z}}\right) .
$$

We say that the $K$-theory of $\mathbb{Z}[\Gamma]$ reduces to finite groups of $\Gamma$ if $\mathcal{A}$ is an isomorphism.

A. Bartels shows in $[\mathbf{1}]$ that $\mathcal{A}$ is a split injection and its cokernel is built from the nil $K$-groups of the rings $\mathbb{Z}[G]$, where $G$ runs over the finite subgroups of $\Gamma$ and other types of nil $K$-groups. By our results, these nil $K$-groups rarely vanish

in higher $K$-theory, hence, in principle, higher $K$-theory does not reduce to finite groups.

\section{References}

[1] A. C. Bartels, On the domain of the assembly map in algebraic $K$-theory, Algebr. Geom. Topol. 3 (2003), 1037-1050.

[2] A. C. Bartels, T. Farrell, L. Jones and H. Reich, On the isomorphism conjecture in algebraic $K$-theory, Topology 43 (2004), 157-213.

[3] A. C. Bartels, W. Lück and H. Reich, The $K$-theoretic Farrell-Jones Conjecture for hyperbolic groups, preprint 813 in http://www.math.uiuc.edu/ K-theory/.

[4] A. C. Bartels and H. Reich, On the Farrell-Jones conjecture for higher algebraic K-theory, J. Amer. Math. Soc. 18 (2004), 501-545.

[5] H. Bass, Algebraic K-Theory, W. A. Benjamin, Inc., New York, 1968.

[6] H. Bass, Some problems in "classical" algebraic $K$-theory, in Algebraic $K$ Theory, II: "Classical" Algebraic K-Theory and Connections with Arithmetic, Lecture Notes in Math. 342 (1973), 3-73.

[7] H. Bass and M. P. Murthy, Grothendieck groups and Picard groups of abelian group rings, Ann. of Math. 86 (1967), 16-73.

[8] E. Berkove, F.T. Farrell, D. Juan-Pineda and K. Pearson, The Farrell-Jones isomorphism conjecture for finite covolume hyperbolic actions and the algebraic K-theory of Bianchi groups, Trans. Amer. Math. Soc. 352 (2000), 56895702.

[9] E. Berkove, D. Juan-Pineda and K. Pearson, The lower algebraic $K$-theory of Fuchsian groups, Comment. Math. Helv. 76 (2001), 339-352. 
[10] J.F. Davis and W. Lück, Spaces over a category and assembly maps in isomorphism conjectures in $K$ - and $L$-theory, K-Theory 15 (1998), 201-252.

[11] F.T. Farrell, The obstruction to fibering a manifold over a circle, Indiana Univ. Math. J. 21 (1971/1972), 315-346.

[12] F.T. Farrell, The nonfiniteness of Nil, Proc. Amer. Math. Soc. 65 (1977), 215-216.

[13] F.T. Farrell and L. Jones, Isomorphism conjectures in algebraic $K$-theory, $J$. Amer. Math. Soc. 6 (1993), 249-297.

[14] M. Gromov, Hyperbolic groups in Essays in Group Theory, Math. Sci. Res. Inst. Publ. 8, 75-263, Springer-Verlag, New York, 1987.

[15] D. Guin-Waléry and J.L. Loday, Obstruction à l'excision en $K$-théorie algébrique, in Algebraic K-Theory (Proc. Conf., Northwestern Univ., Evanston, Ill., 1980), pp. 179-216, Lecture Notes in Math. 854, SpringerVerlag, New York, 1981.

[16] D. Harmon, $N K_{1}$ of finite groups, Proc. Amer. Math. Soc. 100 (1987), 229232.

[17] D. Juan-Pineda and I. Leary, On classifying spaces for the family of virtually cyclic subgroups, Contemp. Math. 407, 135-145, Amer. Math. Soc., Providence, RI, 2006.

[18] D. Juan-Pineda and S. Prassidis, On the nil groups of Waldhusen nils, Topology Appl. 146/147 (2005), 489-499.

[19] W. van der Kallen and J. Stienstra, The relative $K_{2}$ of truncated polynomial rings, J. Pure and Appl. Algebra 34 (1984), 277-289.

[20] I. Ortiz, The lower algebraic $K$-theory of $\Gamma_{3}, K$-Theory 32 (2004), 331-355.

[21] D. Quillen, Higher algebraic K-theory. I, in Algebraic K-Theory, I: Higher K-Theories (Proc. Conf., Battelle Memorial Inst., Seattle, Wash., 1972), pp. 85-147, Lecture Notes in Math. 341, Springer-Verlag, New York, 1973.

[22] T. Vorst, Localization of the $K$-theory of polynomial extensions, Math. Ann. 244 (1979), 33-53.

[23] C. Weibel, The $K$-book: An introduction to algebraic $K$-theory, available at http://math.rutgers.edu/ weibel/Kbook.html.

Daniel Juan-Pineda daniel@matmor.unam.mx

Instituto de Matemáticas, Unidad Morelia

Universidad Nacional Autónoma de México

Campus Morelia

Apartado Postal 61-3 (Xangari)

Morelia, Michoacán

MEXICO 58089

This article is available at http://intlpress.com/HHA/v9/n2/a3 